\title{
Breast cancer proteomics reveals a positive correlation between glyoxalase 1 expression and high tumor grade
}

\author{
MIGUEL A. FONSECA-SÁNCHEZ ${ }^{1}$, SERGIO RODRÍGUEZ CUEVAS ${ }^{2}$, GUILLERMO MENDOZA-HERNÁNDEZ ${ }^{3}$, \\ VERONICA BAUTISTA-PIÑA ${ }^{2}$, ELENA ARECHAGA OCAMPO ${ }^{4}$, ALFREDO HIDALGO MIRANDA ${ }^{5}$, \\ VALERIA QUINTANAR JURADO ${ }^{5}$, LAURENCE A. MARCHAT ${ }^{6}$, ELIZBETH ÁLVAREZ-SÁNCHEZ ${ }^{1}$, \\ CARLOS PÉREZ PLASENCIA ${ }^{7,8}$ and CÉSAR LÓPEZ-CAMARILLO ${ }^{1}$
}

\begin{abstract}
${ }^{1}$ Genomics Sciences Program, Oncogenomics and Cancer Proteomics Laboratory, Autonomous University of Mexico City, 03100 Mexico City; ${ }^{2}$ Institute of Breast Diseases-FUCAM, 04980 Mexico City; ${ }^{3}$ Biochemistry Department, Medicine Faculty, Autonomous National University of Mexico, Mexico City; ${ }^{4}$ Carcinogenesis Laboratory, National Institute of Cancerology, 14080 Mexico City; ${ }^{5}$ National Institute of Genomic Medicine, Mexico City; ${ }^{6}$ Biotechnology Program, Institutional Program of Molecular Biomedicine, National School of Medicine and Homeopathy of the National Polytechnic Institute, 07320 Mexico City; ${ }^{7}$ Massive Sequencing Unit, National Institute of Cancerology, 14080 Mexico City;

${ }^{8}$ Genomics Laboratory, FES-I, UBIMED, National Autonomous University of Mexico, 54090 Mexico City, Mexico
\end{abstract}

Received January 14, 2012; Accepted March 5, 2012

DOI: $10.3892 /$ ijo.2012.1478

\begin{abstract}
Breastcanceris the neoplasia with the highest incidence in women worldwide. Proteomics approaches have accelerated the discovery of diagnostic and prognostic biomarkers. Here, we compared the proteomic profiles of breast tumors versus non-tumoral tissues in order to identify modulated proteins, which could represent potential markers associated to clinical features. By two-dimensional electrophoresis, we detected 28 differentially expressed proteins. Among these, 21 proteins were up-regulated and 7 were down-regulated in tumors $(\mathrm{p}<0.05)$. Proteins were identified using LC/ESI-MS/MS tandem mass spectrometry. One protein was identified as glyoxalase 1 (GLO1), an enzyme involved in detoxification of methylglyoxal, a cytotoxic product of glycolysis. GLO1 overexpression was confirmed by western blot assays in paired normal and tumor breast tissues in clinical stages I-III, and by immunohistochemistry on tissue microarrays (TMA) comprising a cohort of 98 breast tumors and 20 healthy specimens. Results from TMA demonstrated that GLO1 is overexpressed in 79\% of tumors. Interestingly, GLO1 up-regulation correlates with advanced tumor grade $(\mathrm{p}<0.05)$. These findings demonstrate the association of GLO1 overexpression with tumor grade and pointed out for additional studies to establish the importance of GLO1 in breast cancer.
\end{abstract}

Correspondence to: Dr C. López-Camarillo, Genomics Sciences Program, Oncogenomics and Cancer Proteomics Laboratory, Autonomous University of Mexico City, Av. San Lorenzo 290, 03100 Mexico City, Mexico

E-mail: cesar.lopez@uacm.edu.mx

Key words: breast cancer, biomarkers, proteomics, glyoxalase 1, tumor grade

\section{Introduction}

Breast cancer is the neoplasia with the highest incidence and mortality affecting women worldwide with 1.38 million of new cases diagnosed in 2008 (1). In the same period, 185,000 new cases and 40,000 deaths were estimated only in the USA (2). Breast carcinomas represent a heterogeneous group of tumors that are diverse in behavior, outcome, and response to therapy. Despite advances in screening, diagnosis, and therapies, causes of this disease still remain unknown. Current routine clinical management of breast cancer patients relies on clinical and pathological prognostic and predictive factors to support treatment decisions (3). In order to assess prognosis and therapy, oncologic patients are categorized into risk groups based on a combination of prognostic variables (staging based in tumor size, lymph node stage, and extent of tumor spread) and biological prognostic and predictive variables. Biological variables include tumor grade and molecular markers, such as estrogen and progesterone receptors in combination with human epidermal growth factor receptor 2 (HER2, c-erbB2/neu) status (4). Tumor grade is one of the well-established prognostic factors in breast cancer, which represents the morphological assessment of tumor biological characteristics and has been shown to generate information related to the clinical behavior, including aggressiveness, mitotic activity, and overall survival (5). However, molecular markers associated to tumor grade and other prognostic and biological variables are scarce. Because of genetic heterogeneity of breast carcinomas, the role of these classifiers in determining prognosis and evaluating risk in an individual patient is more limited. Therefore, innovative methods to identify novel informative biomarkers according to the molecular features of tumors are required to better assess prognosis and determine the most appropriate treatment for each patient.

High throughput molecular technologies, such as genomewide expression profiling, have been increasingly used to define 
the molecular classification of tumors and assess prognosis and response to therapy in breast cancer $(6,7)$. These genomic studies based on DNA microarrays technology greatly contributed to the understanding of breast cancer heterogeneity and its clinical management, as they provide multigene classifiers represented as molecular fingerprints that have the potential to complement the traditional clinical prognostic and predictive factors $(8,9)$. However, protein-level information is needed for the understanding of cancer proteins function and translation of molecular knowledge into oncological clinical practice. Thus proteomics technologies represent an attractive and complementary approach in biomarkers discovery area. Oncoproteomics has the potential to complement the information generated by genomic profiling because mRNA levels do not always correlate with protein abundance. Contributions of post-translational modifications, such as phosphorylation, acetylation, and glycosylation, are not detectable at mRNA level although they play an important role in the stability, localization and interactions of proteins (10). Moreover, proteins represent more easily accessible therapeutic targets in comparison to nucleic acids. The identification of cancer proteins based in two-dimensional (2-D) electrophoresis, differential in gel electrophoresis (DIGE) and SELDI-TOF strategies has important achievements in the study of breast cancer. Increasing reports using tumor tissues from patients have demonstrated the feasibility of proteomicsbased studies in the identification of novel diagnostic markers and therapeutic targets. For instance, previous studies reported the identification of proteins secreted by estrogen-stimulated cell lines as cathepsin D (11), and the differential expression of keratins between normal and malignant cells (12). Through 2-D techniques, it has been shown that extracellular matrix protein inter- $\alpha$-trypsin inhibitor is negatively regulated in breast cancer (13). Proteomics has also been successfully applied for the identification of proteins involved in the mechanisms of tamoxifen resistance $(14,15)$. In order to contribute to the identification and implementation of novel prognostic markers in breast cancer, we reported here the identification of a set of differentiallyexpressed proteins in normal and tumoral mammary tissues from Mexican women. We show that glyoxalase 1 (GLO1), an enzyme involved in detoxification of methylglyoxal, which is a cytotoxic product of glycolysis, is frequently up-regulated in tumoral mammary tissues and cell lines. In addition, tissue microarrays-based validation of GLO1 expression evidenced for the first time a correlation with advanced tumoral grade and consequently, with an increased proliferative activity of tumors.

\section{Materials and methods}

Cell lines. Cancer cell lines were obtained from the American Type Culture Collection (ATCC). Breast cancer cell lines MCF7, MDA-MB-231, MDA-MB-453 and ZR-75, and lung cancer cell lines A549 and Calu1 were grown in Dulbecco's modified Eagle's medium DMEM-F12 (Gibco, Invitrogen). Cervical cancer cell lines HeLa and SiHa, and colon cancer cell line SW480 were grown in MEM (Gibco, Invitrogen). All culture media were supplemented with $10 \%$ fetal bovine serum (FBS), $100 \mathrm{U} / \mathrm{ml}$ penicillin and $100 \mu \mathrm{g} / \mathrm{ml}$ streptomycin (Gibco, Invitrogen). Cell lines culture were maintained at $37^{\circ} \mathrm{C}$ in a humidified environment with $5 \% \mathrm{CO}_{2}$ and $95 \%$ air. The adherent cells in culture were harvested by trypsinization at $37^{\circ} \mathrm{C}$ for $5-10 \mathrm{~min}$ with
trypsin-EDTA solution (Sigma) and washed with PBS buffer before proteins extraction.

Clinical tumor samples. Human primary tumor tissues and healthy breast biopsies were kindly provided by the Institute of Breast Diseases-FUCAM, Mexico. Tumor and healthy surrounding breast tissues were obtained from the same breast cancer patient after stringent selection following the regulations approved by the FUCAM Ethics Committee, including patient informed consent and anonymatization prior to release for research use. None of the patients recruited in this study received any anti-neoplastic therapy prior to surgery. After tumor resection, specimens were embedded in Tissue-Tek and snap frozen in liquid nitrogen at $-80^{\circ} \mathrm{C}$ until analysis. Pathologist confirmed the existence of at least $80 \%$ tumor cells in specimens. Tumors were classified according to hormonal receptors and HER2 status. For proteomic studies, which were carried out in triplicate to ensure results reproducibility, we carefully selected seven tumors, controlling for histological type (all ductal invasive), classification (all luminal A), and nuclear grade (almost all grade 2). For tissue microarrays (TMA) validation studies, a cohort of 98 breast cancer patients was recruited in this study. Mammary specimens obtained from normal adjacent tissues were conjointly analyzed in order to obtain a master gel, and to minimize the misinterpretation of protein profiles arising from random differences in gene expression of different tumors.

Protein extraction and separation by two-dimensional electrophoresis. Frozen breast tumors and healthy tissues were disrupted using a TissueRuptor (Qiagen) handheld rotor stator homogenizer. Protein samples were extracted from tissues using $500 \mu 1$ TNTE buffer (50 mM Tris- $\mathrm{HCl}$ pH 7.4, $150 \mathrm{mM} \mathrm{NaCl}$, $0.5 \%$ Triton X-100, $5 \mathrm{mM}$ EDTA) in the presence of $40 \mu \mathrm{l} / \mathrm{ml}$ Complete proteases inhibitor cocktail (Roche) at $4^{\circ} \mathrm{C}$. Samples were centrifuged at $14,000 \mathrm{rpm}$ for $5 \mathrm{~min}$ at $4^{\circ} \mathrm{C}$. Supernatant was retrieved and cleaned using the ReadyPrep 2D Clean Up kit (Bio-Rad) according to the manufacturer's protocol. Then, proteins were re-dissolved in $100 \mu \mathrm{l}$ sample buffer $(7 \mathrm{M}$ urea, 2\% CHAPS, $40 \mathrm{mM}$ dithiotreitol (DTT), 0.5\% Triton X-100). Protein concentrations were determined using the Bradford method. For the first-dimension, proteins $(200 \mu \mathrm{g})$ obtained from tumoral and non-tumoral mammary tissues were mixed with $120 \mu \mathrm{l}$ rehydration solution containing $7 \mathrm{M}$ urea, 2\% CHAPS, $40 \mathrm{mM}$ dithiotreitol, $0.5 \%$ ampholines $\mathrm{pH} 3.0-10$, and $0.002 \%$ blue bromophenol and loaded onto $11 \mathrm{~cm}$ ReadyStrip IPG strips (linear pH gradient 4.0-7.0, Bio-Rad). IPG strips were passively hydrated for $16 \mathrm{~h}$ at room temperature. Then, proteins were isoelectrically focused using the Protean IEF Cell (Bio-Rad) in four steps: an initial gradient from 1 to $150 \mathrm{~V}$ for $2 \mathrm{~h}$, followed by a gradual increase up to $8,000 \mathrm{~V}$ for $3 \mathrm{~h}$, for a total of 35,000 Vh. Finally, a hold step at $100 \mathrm{~V}$ was applied. Then, IPG strips were equilibrated for reduction and alkylation in equilibration buffer (6 $\mathrm{M}$ urea, $2 \% \mathrm{SDS}, 0.375 \mathrm{M}$ Tris- $\mathrm{HCl}$ $\mathrm{pH} 8.8,20 \%$ glycerol) with $2 \%$ DTT and $2.5 \%$ iodoacetamide in first and second washes, respectively, for $10 \mathrm{~min}$ at room temperature. For second-dimension, proteins were resolved by $12 \%$ SDS-PAGE. Gels were run in running buffer $(25 \mathrm{mM}$ Tris- $\mathrm{HCl}, 192 \mathrm{mM}$ glycine, $0.1 \% \mathrm{SDS}$ ) at $50 \mathrm{~V}$ for $20 \mathrm{~min}$ and $200 \mathrm{~V}$ until samples reach the bottom of gel. Protein gels were overnight stained with Sypro-Ruby dye (Invitrogen) according 
to manufacturer's protocol; then, images from 2-D gels were documented in a FLA-5100 Fuji Film scanner and adjusted using the Multigauge software. PD-Quest Advanced software version 8.0 (Bio-Rad) was used for comparative analyses of images corresponding to proteins obtained from tumor and non-tumor biopsies. For 2-D spots selection, images of gels from normal and tumor tissues were used to create a match-set. Spots were detected and automatically matched to a master gel selected by the PDQuest software. The spot detection, spot boundary tool and matching were manually edited. The spots were checked and manually added to the master gel to allow matching of unique spots present in the individual gels. Spot quantities were normalized to remove variations non-related to expression changes. The criterion of a differential expression of any particular protein between the subset of tissues was set as at least a 2-fold change in spot volume between matched sets in triplicates. All these spots were selected for subsequent analysis.

Tandem mass spectrometry (LC/ESI-MS/MS). Protein spots were excised from Sypro-Ruby stained gels, washed with $50 \%(\mathrm{v} / \mathrm{v})$ methanol, $5 \%(\mathrm{v} / \mathrm{v})$ acetic acid for $2 \mathrm{~h}$ and then, with deionized water 3 times, $10 \mathrm{~min}$ each. The destained gels were soaked for $10 \mathrm{~min}$ in $100 \mathrm{mM}$ ammonium bicarbonate, cut into small pieces, completely dehydrated with $100 \%$ acetonitrile and vacuum-dried. In gel digestion was performed by adding $30 \mu \mathrm{l}$ of modified porcine trypsin solution (Promega, Madison, WI, USA) containing $20 \mathrm{ng} / \mu \mathrm{l}$ in $50 \mathrm{mM}$ ammonium bicarbonate followed by overnight incubation at room temperature. Peptides were extracted with $50 \%(\mathrm{v} / \mathrm{v})$ acetonitrile, $5 \%(\mathrm{v} / \mathrm{v})$ formic acid twice for $30 \mathrm{~min}$, and each time with sonication. The volume of the extracts was reduced by evaporation in a vacuum centrifuge and then adjusted to $20 \mu \mathrm{l}$ with $1 \%$ (v/v) formic acid.

Mass spectrometric analysis was carried out on a $3200 \mathrm{Q}$ TRAP hybrid tandem mass spectrometer (Applied Biosystems/ MDS Sciex, Concord, ON, Canada), equipped with a nano electrospray ion source (NanoSpray II) and a MicroIonSpray II head. The instrument was coupled on-line to a nanoAcquity Ultra Performance LC system (Waters Corporations, Milford, MA). Mass calibration of the hybrid triple quadrupole linear ion trap spectrometer was done with polypropylene glycol standard solutions. The instrument was then tuned and tested using [Glu1]fibrinopeptide B (Sigma). Samples were desalted by injection onto a Symmetry $\mathrm{C}_{18}$ UPLC trapping column $(5 \mu \mathrm{m}, 180 \mu \mathrm{m}$ x $20 \mathrm{~mm}$, Waters Corporations) and washed with $0.1 \%$ formic acid in $100 \%$ Milli Q water at a flow rate of $15 \mu \mathrm{l} / \mathrm{min}$. After $3 \mathrm{~min}$, the trap column was switched in-line with the analytical column. Peptides were separated on a $\mathrm{BEH}, \mathrm{C}_{18}$ UPLC column (1.7 $\mu \mathrm{m}, 75 \mu \mathrm{m} \times 100 \mathrm{~mm}$, Waters Corporations) equilibrated with $2 \%$ acetonitrile, $0.1 \%$ formic acid using a linear gradient of $2-70 \%$ acetonitrile, $0.1 \%$ formic acid over a 60 -min period, at a flow rate of $0.25 \mu \mathrm{l} / \mathrm{min}$. Spectra were acquired in automated mode using information dependent acquisition (IDA), which involves switching from MS to MS/MS mode on detection of +2 to +4 charged species. The precursor ions were fragmented by collisionally-activated dissociation (CAD) in the Q2 collision cell. Collision voltages were automatically adjusted based upon the ion charge state and mass using rolling collision energy. Other instrument operation conditions were as described previously (16).
Data interpretation and protein identification were performed with the MS/MS spectra data sets using the MASCOT search algorithm (version 1.6b9, Matrix Science, London, UK available at http://www.matrixscience.com). Searches were conducted using the Homo sapiens subset of the National Center for Biotechnology Information non-redundant database (NCBInr, http://www.ncbi.nih.gov). Trypsin was used as the specific protease and one missed cleavage was allowed with tolerances of 0.5 Da for the precursor and 0.3 Da for the fragment ion masses. Carbamidomethyl-cysteine and methionine oxidation were used as the fixed and variable modifications, respectively. A protein 'hit' was accepted as a valid identification when at least two MS/MS spectrum matched at the $95 \%$ level of confidence $(\mathrm{p}<0.05)$. Ion score is $-10^{*} \log (\mathrm{P})$, where $\mathrm{P}$ is the probability that the observed match is a random event. The threshold ion score in the above conditions was 41 for $\mathrm{p}<0.05$.

Western blot assays. GLO1 expression in breast tumor and normal tissues, and cancer cell lines from breast (MCF7, MDA-MB-231, MDA-MB-453, ZR-75), lung (A549, Calu1), cervix (SiHa, HeLa), and colon (SW480) was evaluated by western blot assays. Confluent cell cultures and tissues samples were lysed using SDS-buffer containing a complete mini-protease inhibitor cocktail tablet (Roche Molecular Biochemicals). Total protein concentration was estimated using the Bradford protein assay (Bio-Rad). Protein extracts $(35 \mu \mathrm{g})$ were separated by $15 \%$ SDS-PAGE and electrotransferred to a PVDF membrane (Millipore). Membranes were probed with $0.5 \mu \mathrm{g} / \mathrm{ml}$ anti-GLO1 monoclonal antibody (AbCam ab85420) in $5 \%$ non-fat dry milk and $0.05 \%$ Tween-20 in PBS pH 7.4 overnight at $4^{\circ} \mathrm{C}$. For detection, membranes were incubated with peroxidase-conjugated anti-mouse (Molecular Probes, Invitrogen) secondary antibodies $(1: 5,000)$ in $5 \%$ non-fat dry milk and $0.05 \%$ Tween-20 in PBS pH 7.4 and immunocomplexes were developed using the ECL chemiluminescence system (Amersham Pharmacia Biotech). Finally, membranes were subjected to strip and re-blot using antibodies raised against $\beta$-actin as control.

Immunohistochemistry on tissue microarrays. High throughput analysis of 98 breast tumors and 20 normal mammary tissues was performed using a home-made tissue microarray (TMA, Tissue Microarrayer ATA100 Chemicon) and immunohistochemistry. Briefly, sections of $0.3 \mathrm{~mm}$ thickness from both tumoral and non-tumoral specimens were deparafinized in xylene and rehydrated in graded ethanol and water. Antigen unmasking was performed using $0.01 \mathrm{M}$ sodium citrate $\mathrm{pH} 6.0$ for $10 \mathrm{~min}$. Endogenous peroxidases were removed using $3 \%$ hydrogen peroxide for $30 \mathrm{~min}$ at room temperature in humid chamber. Samples were blocked for $1 \mathrm{~h}$ at room temperature with $1 \%$ albumin. Then, sections were incubated overnight at $4^{\circ} \mathrm{C}$ with anti-GLO1 antibodies (1:50) followed by incubation with universal secondary antibodies for $15 \mathrm{~min}$ and detection using Trek avidin-HRP for $10 \mathrm{~min}$ and DAB (3,3'-diaminobenzidine tetrahydrochloride)-substrate chromagen solution for $15 \mathrm{sec}$ (Detection System, StarTrek, HRP universal kit). Nuclei were stained with Mayer's hematoxylin before imaging, and slides were mounted with Permont reactive. The staining level of GLO1 protein was scored as negative (0), weak (1), moderate (2), and strong (3). 
Table I. Clinical features of breast tumors analyzed by two-dimensional gels in this study.

\begin{tabular}{|c|c|c|c|c|c|c|c|c|c|}
\hline Patient & Age & ER & PR & HER2 & Tumor size (mm) & Histology & Classification & Clinical stage (TNM) & Tumor grade \\
\hline 115 & 45 & + & + & - & 10 & Ductal invasive & Luminal A & IIA & 2 \\
\hline 116 & 92 & + & - & - & 80 & Ductal invasive & Luminal A & IIIB & 2 \\
\hline 125 & 57 & + & - & - & 25 & Ductal invasive & Luminal A & IIB & 2 \\
\hline 129 & 53 & + & + & - & 13 & Ductal invasive & Luminal A & IIA & Unknown \\
\hline 133 & 61 & + & + & - & 17 & Ductal invasive & Luminal A & IIIA & 2 \\
\hline 151 & 64 & + & + & - & 25 & Ductal invasive & Luminal A & IIA & 2 \\
\hline 158 & 56 & + & + & - & 23 & Ductal invasive & Luminal A & IIA & 2 \\
\hline
\end{tabular}

ER, estrogen receptor; PR, progesterone receptor; HER2, human epidermal growth factor receptor 2.

Statistical analysis. The mean of logarithmic ratios method was used for normalization. It calculates the normalization factor of a gel by calculating the mean of all $\log$ ratios of all matched spots (master gel versus gel). Spots down- and up-regulated were analyzed with quantity-test for 2.0-fold and with the t-test with intervals of $95 \%$. For clinical correlation and GLO1 expression, a $2 \times 2$ and $3 \times 3 \chi^{2}$ test was utilized to assess significance among categorical variables. Tumor grade variable was determined as $\mathrm{p}<0.01$.

\section{Results}

Proteomic identification of deregulated proteins in sporadic ductal breast tumors. To identify differentially-expressed proteins in sporadic breast cancer, we compared the proteomic profiles from seven ductal invasive carcinomas and five nontumor mammary tissues. Clinical features of breast tumors including hormonal receptor status, tumor size, histology, clinical stage, and tumoral grade are summarized in Table I. Protein extracts were analyzed using IPG strips with a $\mathrm{pH}$ 4.0-7.0 linear gradient, which resulted in a better separation of protein spots. Fig. 1A shows two representative two-dimensional electrophoresis gels from non-tumor (upper panel) and tumor (lower panel) tissues. The proteomic profiles obtained for the total of specimens were highly reproducible (data not shown). Densitometric analysis of 17 selected spots from healthy and tumor tissues evidenced the differential protein expression between both proteomic profiles (Fig. 1B).

The comparative analysis of images obtained from 2-D gels using the PD-Quest software (Bio-Rad), allowed the detection of 28 differentially-expressed proteins in the set of biopsies ( $>2.0$-fold change, $\mathrm{p}<0.05)$. Among these, 21 proteins were up-regulated and 7 were down-regulated in tumors. After spots picking, trypsin in-gel digestion, LC/ESI-MS/MS tandem mass spectrometry analysis and NCBI database search, 17 modulated proteins were identified.

The identity and function of proteins, Mascot scores, sequence coverage, and MS/MS peptide sequences (ion score) are summarized in Table II. These included proteins with redox and detoxification functions (spot 1, GLO1; spot 3, PRDX6; spot 14, SOD1; spot 12, DJ-1), a stress-associated protein (spot 5, HSP27), a protein involved in intermediary metabolism (spot 4, ECHS1), two molecular chaperones (spot 2, TBCA; spot 15, HSP70), a exocytosis protein (spot 17, ANXA1), a signaling transduction factor (spot 13, RhoGDI-2), a neural survival factor (spot 11, DCD), a platelet aggregation protein (spot 10, FGB), and a cell proliferation and metastasis protein (spot 6, nm23), among others. Notably, all these proteins have been previously related to breast cancer development, progression, and invasion (17-28).

GLO1 is overexpressed in breast tumors and in diverse cancer cell lines. One of the most abundant up-regulated proteins corresponded to glyoxalase 1 GLO1 [lactoylglutathione lyase (EC 4.4.1.5)]\}, which participates in detoxification of methylglyoxal, a cytotoxic bioproduct of glycolysis, by catalyzing the conversion of toxic $\alpha$-oxo-aldehydes into the corresponding $\alpha$-hydroxy acids using L-glutathione (GSH) as cofactor (28). Although GLO1 expression has been reported in several human cancer types, its clinical relevance in breast cancer is poorly understood.

To analyze the expression of GLO1 in breast tumors, we first analyzed the individual proteomic profiles obtained from tumor and non-tumor breast tissues and observed a consistent up-regulation of this protein (Fig. 2A and B). GLO1 overexpression was further validated by western blot assays in a panel of six paired tumor and non-tumor mammary tissues grouped by clinical stages (I-III). Results showed that GLO1 was up-regulated in $50 \%$ of tumor specimens with a higher expression in clinical stage III (Fig. 2C and D). In addition, we investigated the GLO1 expression in four breast cancer cell lines (MCF7, MDA-MB231, MDA-MB-453, ZR-75), two lung cancer cell lines (A549, Calu1), two cervical cancer cell lines (SiHa, HeLa), and a colon cancer cells (SW480) by western blot analysis. Results showed that GLO1 expression was variable in the different cell lines (Fig. 2E and F). In breast cancer MCF7 and MDA-MB-231 cell lines, GLO1 was abundantly expressed in comparison with MDA-MB-453 and ZR-75. In addition, HeLa cervix tumor cells exhibited high levels of GLO1 expression. In contrast, lung cancer A549 and Calu1, cervical cancer SiHa and colon cancer SW480 cells exhibited low GLO1 expression levels. These data indicate that GLO1 overexpression is not confined for breast tumors and suggested that GLO1 aberrant expression is frequent in some human cancers.

GLOI overexpression correlates with tumor grade. In order to validate the 2 -D results initially obtained from a limited set of 
A
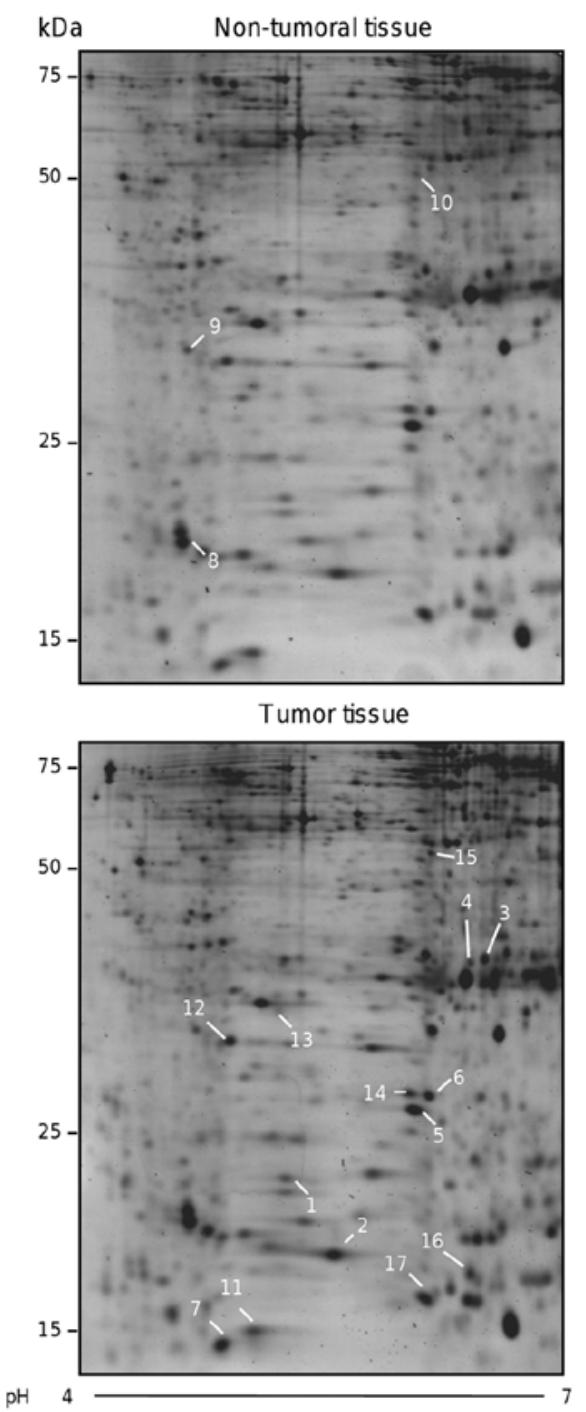

B

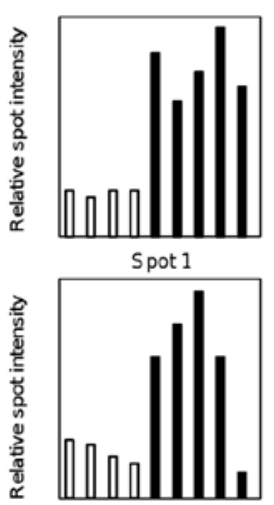

Spot 5

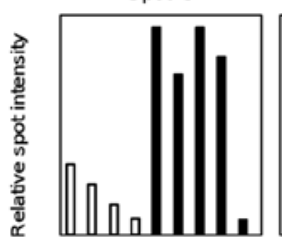

Spot 9
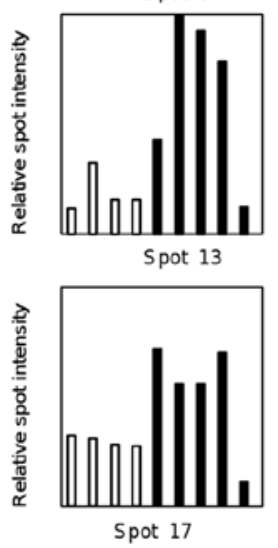

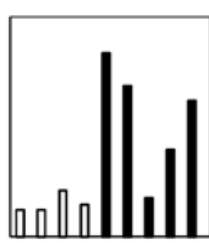

Spot 2

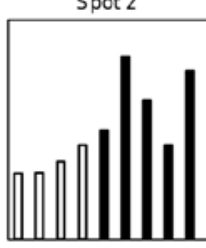

Spot 6

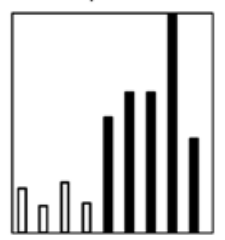

Spot 10

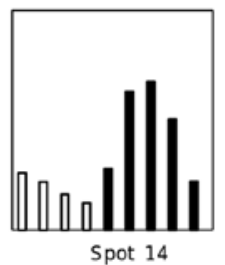

$\square$ Non-tumoral tissue

Tumor tissue

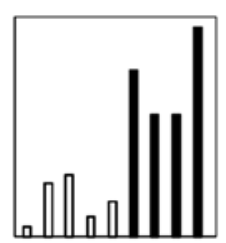

Spot 3

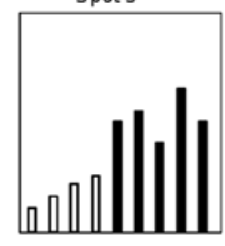

Spot 7

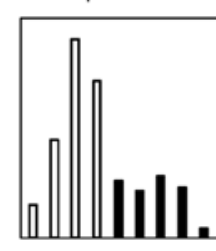

Spot 11

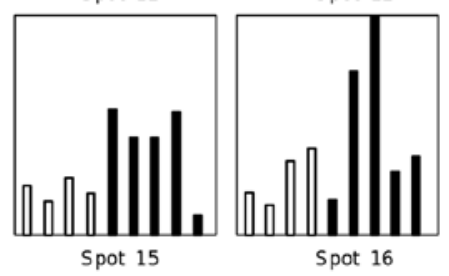

Spot 8

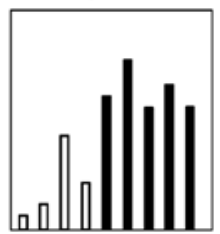

pot 4

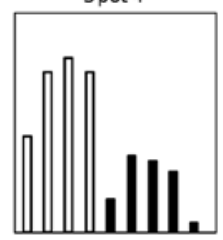

\section{(n)}

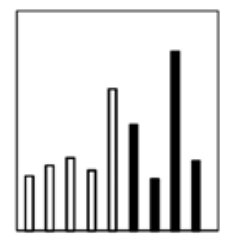

\section{(1)}
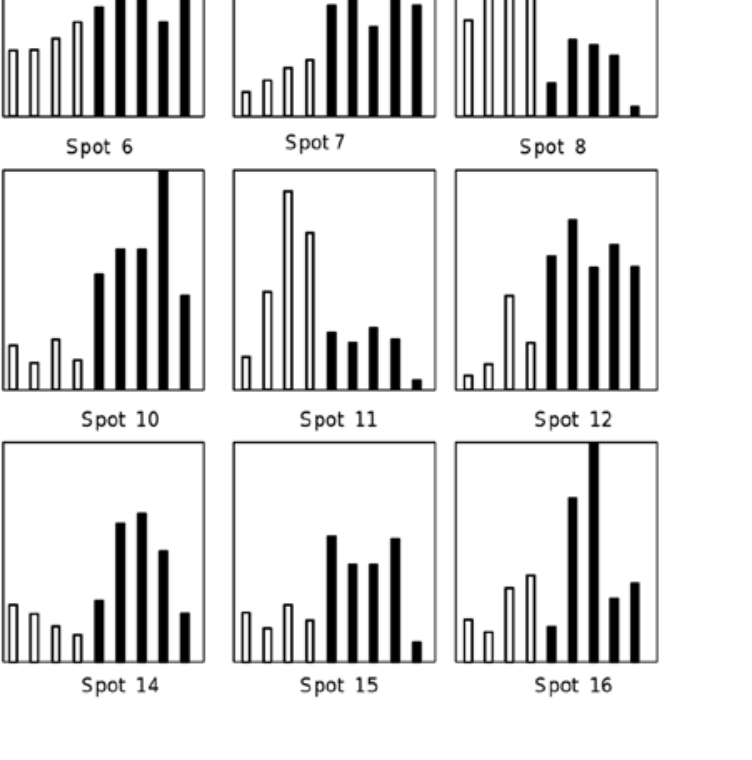

Figure 1. Proteomic analysis of breast tumor and non-tumor tissues. (A), Representative cropped 2-D gel images of protein profiles showing differentially expressed protein spots (marked with numbers) in tumor and non-tumor tissues in the $\mathrm{pH}$ range of 4.0-7.0. (B), Densitometric analysis of selected protein spots from gels in (A) modulated in the eight tumors and four non-tumor tissues. The histograms show relative protein spot quantities. Spots were determined as significantly differentially-expressed according to Student's t-test $(\mathrm{p}<0.05)$.

biopsies, we analyzed GLO1 expression in a panel of 98 breast tumors and 20 healthy specimens by immunohistochemistry using TMA. Clinical characteristics of breast tumors analyzed are summarized in Table III. Results indicated that all the healthy specimens showed a weak staining (0-1 score) for GLO1 protein, which representing the basal levels of GLO1 expression. Interestingly, we observed that about $79 \%$ of the tumor tissues on TMA sections showed from moderate to strong intensity staining by anti-GLO1 monoclonal antibodies (Fig. 3 and Table IV). In previous studies no correlation between GLO1 expression and clinicopathological data was reported, thus here we established its clinical relevance by searching for an association between GLO1 expression and clinical characteristics of patients, mainly tumor size, nodal status, clinical stage, hormonal receptors, the presence of lymph and tumor grade. Results evidenced that there was a statistically significant correlation between GLO1 expression and advanced tumor grade (Table $\mathrm{V}, \mathrm{p}<0.05)$.

\section{Discussion}

Previous transcriptomic studies using DNA microarrays have allowed the identification of a large number of genes that are differentially-expressed between human normal and tumor mammary tissues (6-9). However, few of those identified genes have been translated into valid protein markers that can help in diagnosis and effective treatment $(29,30)$. Therefore, proteinlevel information represents a complementary approach to the understanding of cancer proteins function and translation of molecular knowledge into oncological clinical practice. In particular, oncoproteomics technologies offer an attractive and complementary approach in biomarkers discovery. In this study, we performed traditional two-dimensional gel electrophoresis in order to contribute to the identification of novel potential markers with clinical value in breast cancer in Mexican women. The proteomic profiles exhibited similitudes and differences with 


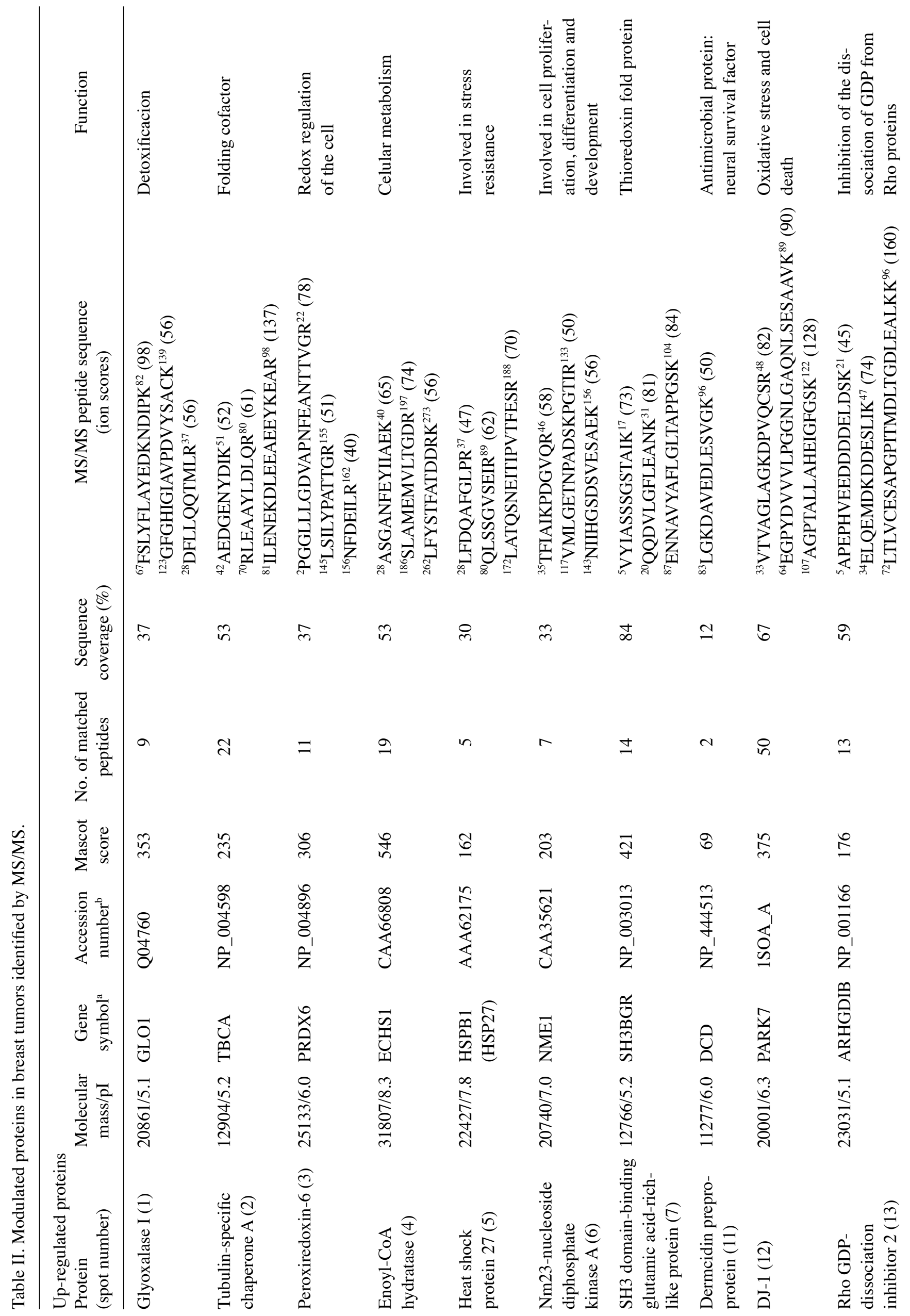




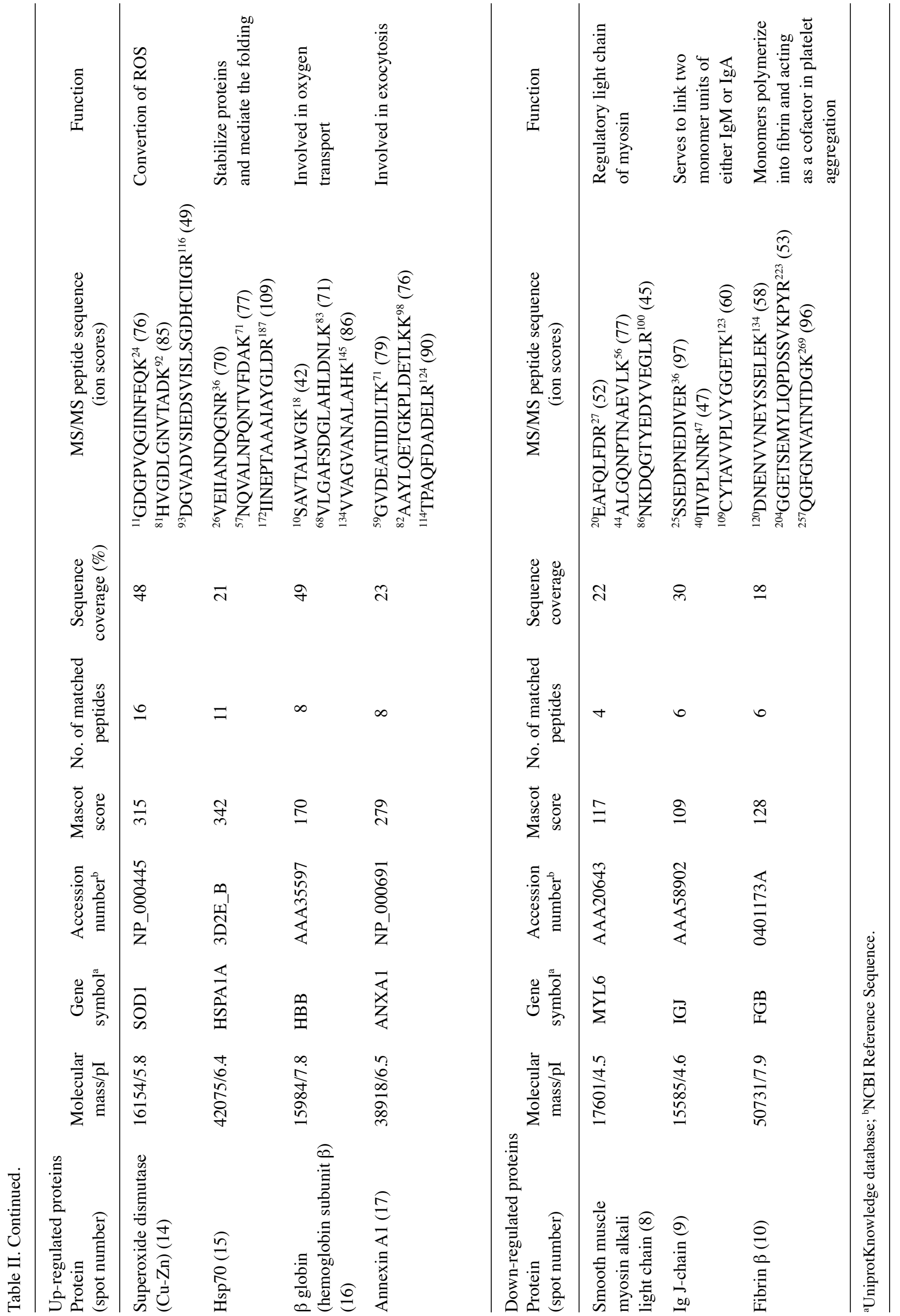


A

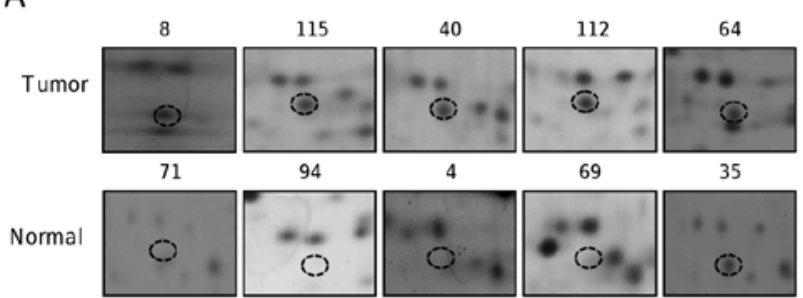

C

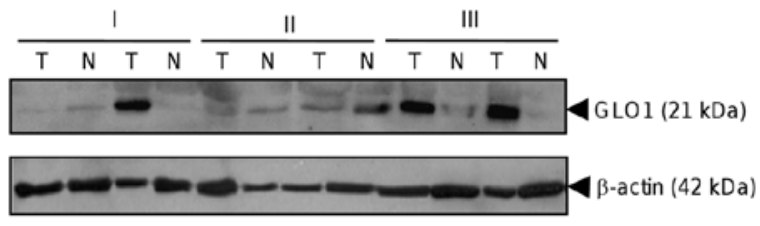

$\mathrm{E}$

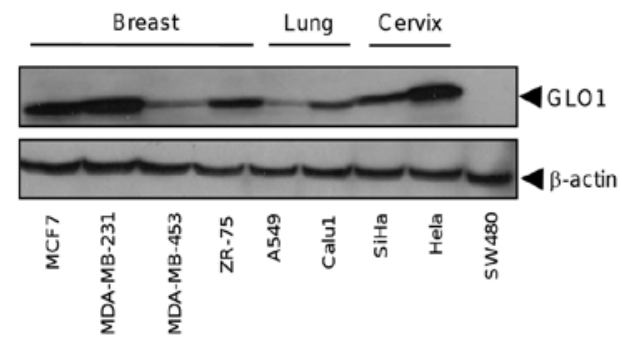

B

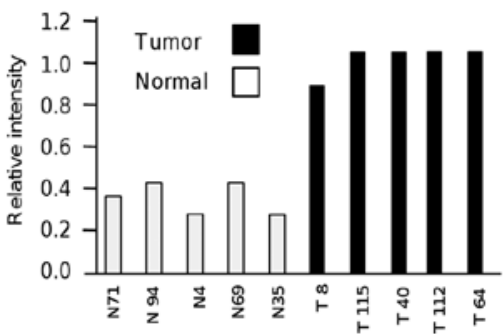

D

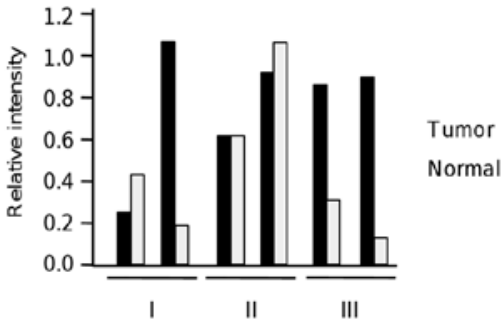

$\mathrm{F}$

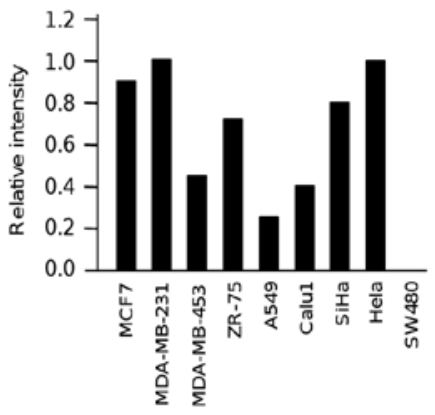

Figure 2. Expression of glyoxalase 1 in tumor and healthy adjacent mammary tissues. (A), Cropped representative 2-D gel images of GLO1 spots (denoted as discontinuous circles) in four healthy and seven tumor tissues. (B), Densitometric quantification of GLO1 spots in each individual tissue sample depicted in (A). Each bar graph represents the specimen number as normal (N) and tumoral (T). (C), GLO1 expression in six matched mammary biopsies. Protein lysates from (T) tumor and $(\mathrm{N})$ normal were analyzed by western blot assay using anti-GLO1 monoclonal antibodies. $\beta$-actin was probed as an internal loading control. Roman numbers indicates the clinical stage of each specimen. (D), Densitometric quantification of GLO1 bands depicted in (C). (E), Western blot assay for GLO1 expression in nine human cancer cell lines. Protein lysates from breast (MCF7, MDA-MB-231, MDA-MB-453, ZR-75), lung (A549, Calu1), cervix (SiHa, HeLa), and colon (SW480) cancers were separated by 12\% SDS-PAGE, transferred to nylon membranes and blotted with anti-GLO1 monoclonal antibodies. $\beta$-actin was probed as an internal loading control. (F), Densitometric quantification of GLO1 bands depicted in (E).

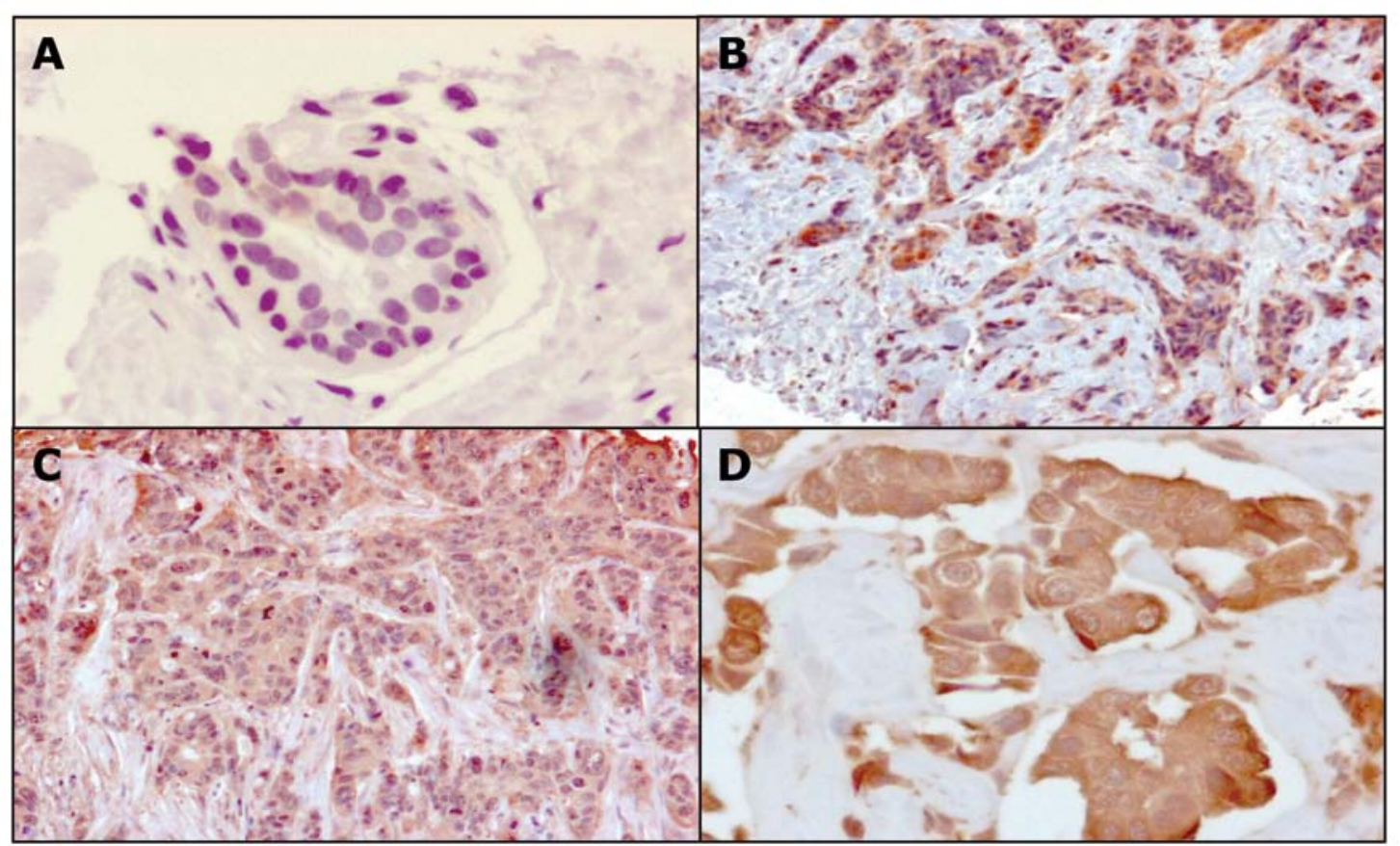

Figure 3. Representative photographs of immunohistochemical detection of GLO1 in tissue microarrays. (A), Normal mammary tissue staining with anti-GLO1 antibodies. GLO1 expression in tumor grade 1 (B), grade 2 (C) and grade 3 (D) breast tissues. Magnification, x400. 
Table III. Characteristics of breast tumors analyzed in tissue microarrays.

\begin{tabular}{|c|c|c|}
\hline Characteristics & Total & $(\%)$ \\
\hline No. of patients & 98 & $(100)$ \\
\hline \multicolumn{3}{|l|}{ Age (years) } \\
\hline $30-39$ & 6 & $(6.12)$ \\
\hline $40-59$ & 48 & $(48.97)$ \\
\hline$>60$ & 27 & $(27.55)$ \\
\hline Unknown & 17 & $(17.34)$ \\
\hline Mean & 54.78 & \\
\hline Primary surgery & 98 & $(100)$ \\
\hline \multicolumn{3}{|l|}{ Histological type } \\
\hline Ductal in situ & 1 & $(1.02)$ \\
\hline Ductal invasive & 58 & $(59.18)$ \\
\hline Lobular invasive & 8 & $(8.16)$ \\
\hline Other & 15 & $(15.30)$ \\
\hline Unknown & 16 & $(16.32)$ \\
\hline \multicolumn{3}{|l|}{ Clinical stage } \\
\hline $0-\mathrm{I}$ & 14 & $(14.28)$ \\
\hline II & 56 & $(57.14)$ \\
\hline III & 9 & $(9.81)$ \\
\hline Unknown & 19 & (19.38) \\
\hline \multicolumn{3}{|l|}{ Tumor size (cm) } \\
\hline$<2$ & 9 & $(9.18)$ \\
\hline$\geq 2$ & 49 & $(50)$ \\
\hline$\geq 4$ & 21 & $(21.42)$ \\
\hline Unknown & 19 & (19.38) \\
\hline \multicolumn{3}{|l|}{ Nodal status } \\
\hline No & 60 & $(61.22)$ \\
\hline$\geq \mathrm{N} 1$ & 19 & (19.38) \\
\hline Unknown & 19 & $(19.38)$ \\
\hline \multicolumn{3}{|l|}{ Metastasis } \\
\hline No detected & 98 & $(100)$ \\
\hline \multicolumn{3}{|l|}{ Hormonal receptors } \\
\hline ER & 54 & $(55.10)$ \\
\hline PR & 46 & (46.93) \\
\hline Her2 & 15 & $(15.30)$ \\
\hline Absent & 14 & (14.28) \\
\hline \multicolumn{3}{|l|}{ SBR } \\
\hline 5 & 5 & $(5.10)$ \\
\hline 6 & 33 & (33.67) \\
\hline $7-9$ & 25 & $(25.51)$ \\
\hline Unknown & 35 & $(35.71)$ \\
\hline \multicolumn{3}{|l|}{ Nuclear grade } \\
\hline 1 & 6 & $(6.12)$ \\
\hline 2 & 56 & (57.14) \\
\hline 3 & 16 & (16.32) \\
\hline Unknown & 20 & $(20.40)$ \\
\hline
\end{tabular}

Table IV. Inmunohistochemical analysis of GLO1 expression in breast tumor and non-tumor tissues using tissue microarrays.

\begin{tabular}{lcc}
\hline Staining scores & Tumors $(\mathrm{n}=98)$ & Normal $(\mathrm{n}=20)$ \\
\hline Negative & 0 & 0 \\
Weak & $21(21.4 \%)$ & $20(100 \%)$ \\
Moderate & $43(43.87 \%)$ & 0 \\
Strong & $34(34.69 \%)$ & 0 \\
\hline
\end{tabular}

Table V. Correlation of GLO1 expression with tumor grade in breast tumors.

\begin{tabular}{lrcrc}
\hline & \multicolumn{3}{c}{ GLO1 expression } & \\
\cline { 2 - 4 } Tumoral grade & Weak & Moderate & Strong & Total \\
\hline 1 & 2 & 4 & 0 & 6 \\
2 & 11 & 31 & 14 & 56 \\
3 & 0 & 2 & 14 & 16 \\
Total & 13 & 37 & 28 & 78 \\
\hline
\end{tabular}

those found previously by several authors in other geographic populations. Coincidences include the overexpression of $\mathrm{nm} 23$, perodoxin-6, Hsp27, and DJ-1 proteins, which have been previously reported in several human cancer types. For instance, it has been shown that serum levels of DJ-1 are increased in breast cancer patients in comparison with healthy subjects. In addition DJ-1 is a recognized androgen receptor coactivator (31), and is overexpressed in human prostate cancer (32). Moreover, DJ-1 has been considered as a prognostic value in ovarian carcinoma with effusions.

Particularly, we observed the overexpression of GLO1 in breast tumor tissues, as it has been previously reported in several human cancers. The glutathione-dependent glyoxalase system, composed of GLO1 and GLO2 enzymes is involved in the detoxification of methylglyoxal, a side toxic product of glycolysis that may react with DNA, RNA, and proteins and cause cell apoptosis if accumulated (28). This endogenous metabolite causes glycation of nucleotides to form advanced glycation end-products (AGEs) which induce single-strand breaks, DNA-protein cross-link and cytotoxicity. Alterations in GLO1 enzyme expression and activity have been previously reported in human cancers (33-37). Sakamoto et al showed that GLO1 is involved in apoptosis resistance to antitumor agents in human leukemia cells, and that use of GLO1 inhibitors sensitizes cells to chemotherapeutic agents (38). Particularly, the glol gene was amplified in 8/37 (22\%) of breast tumors (39). These events may reflect a response of tumor cells to elevated cellular methylglyoxal stress associated with glycolytic adaptations referred as the 'Warburg' effect (40).

In the studies mentioned above, no correlation with clinicopathological data was done, thus clinical relevance of GLO1 overproduction was unknown until the present work. Data from 
immunohistochemistry on TMA confirmed that GLO1 protein was overexpressed in most breast tumors, suggesting that gene expression regulation mechanisms may be occurring at RNA and protein level. In addition, they evidenced the up-regulation of GLO1 enzyme in sporadic ductal breast cancer and established a strong correlation with tumor grade 3 . Notably, our findings from 2-D analysis of breast tumors and non-tumor tissues, and the validation in a cohort of 98 patients, showed that GLO1 overexpression does not correlate with hormonal receptor status, neither HER2-positive tumors. In fact, all the tumors analyzed by conventional 2-D electrophoresis were HER2-negative. In contrast, Zhang et al reported that GLO1 was exclusively overexpressed in HER2-positive breast tumors by using TMA (17). These differences may be due to genetic differences between Singapore and Mexico women populations studied in these reports, which could represent the high heterogeneity of breast cancer.

GLO1 overexpression was positively correlated with high tumor grade. Grade refers to a 'score' that tells us how different the cancer cell appearance and growth patterns are from those of normal healthy cells, and it has predictive prognosis value. Implications of these findings are important for patient prognosis, because grade 3 tumors seem to be undifferentiated, aggressive, with loss of tubules and high mitotic activity (41). Unfortunately, 5-year overall survival of patients with grade 3 tumors is only $50 \%$ in comparison with grade 1 and 2 tumors, which is estimated as 90 and $75 \%$, respectively. These findings point out for the potential use of GLO1 as a novel marker for tumor grade with a prognostic value. Additionally, GLO1 could be used to distinguish between aggressive and less aggressive tumors. Intriguingly, we observed the presence of GLO1 inside the nucleus in about $10 \%$ of tumors, which has not been previously reported. These findings suggest a potential nuclear function of GLO1 in detoxification of methylglyoxal to prevent formation of nuclear advanced glycation end-products, allowing cell survival. Overexpression of GLO1 could indicate a defense response of tumor cells to elevated levels of methylglyoxal associated with high glycolytic rates referred as the 'Warburg' effect. Our data suggested that GLO1 up-regulation is a common event in cancer and the potential use of GLO1 inhibitors as useful adjunct anticancer drugs deserve further validation.

\section{Acknowledgements}

The authors gratefully acknowledge the financial support from the National Council of Science and Technology, CONACyT Mexico (grants 112454 and 115306), and The Institute of Science and Technology, ICyT-DF, Mexico (grant no. PIFUTP09269). This work was also supported by UACM (Mexico), and COFAA-IPN (Mexico). We also acknowledge Dr Sonia Labastida Almendaro for support in statistical analysis.

\section{References}

1. Ferlay J, Shin HR, Bray F, Forman D, Mathers C and Parkin DM Estimates of worldwide burden of cancer in 2008: GLOBOCAN 2008. Int J Cancer 127: 2893-2917, 2010.

2. Jemal A, Siegel R, Ward E, Hao Y, Xu J, Murray T and Thun MJ: Cancer Statistics, 2008. CA Cancer J Clin 58: 71-96, 2008.

3. Schnitt SJ: Classification and prognosis of invasive breast cancer: from morphology to molecular taxonomy. Mod Pathol 23: S60-S64, 2010.
4. Rakha EA and Ellis IO: Modern classification of breast cancer: should we stick with morphology or convert to molecular profile characteristics. Adv Anat Pathol 18: 255-267, 2011.

5. Rakha EA, Reis-Filho JS, Baehner F, et al: Breast cancer prognostic classification in the molecular era: the role of histological grade. Breast Cancer Res 12: 207, 2010.

6. Perou CM, Sørlie T, Eisen MB, et al: Molecular portraits of human breast tumours. Nature 406: 747-752, 2000.

7. Murphy N, Millar E and Lee CS: Gene expression profiling in breast cancer: towards individualising patient management. Pathology 37: 271-277, 2005.

8. Correa Geyer F and Reis-Filho JS: Microarray-based gene expression profiling as a clinical tool for breast cancer management: are we there yet? Int J Surg Pathol 17: 285-302, 2009.

9. Tyers M and Mann M: From genomics to proteomics. Nature 422: 193-197, 2003.

10. Bertucci F, Birnbaum D and Goncalves A: Proteomics of breast cancer: principles and potential clinical applications. Mol Cell Proteomics 5: 1772-1786, 2006.

11. Westley B and Rochefort H: A secreted glycoprotein induced by estrogen in human breast cancer cell lines. Cell 20: 353-362, 1980.

12. Trask DK, Band V, Zajchowski DA, Yaswen P, Suh T and Sager R: Keratins as markers that distinguish normal and tumor-derived mammary epithelial cells. Proc Natl Acad Sci USA 87: 2319-2323, 1990.

13. Stein RC and Zvelebil MJ: The application of 2-D gel-based proteomics methods to the study of breast cancer. J Mammary Gland Biol Neoplasia 7: 385-393, 2002.

14. Umar A, Kang H, Timmermans AM, et al: Identification of a putative protein profile associated with tamoxifen therapy resistance in breast cancer. Mol Cell Proteomics 8: 1278-1294, 2009.

15. Besada V, Diaz M, Becker M, Ramos Y, Castellanos-Serra L and Fichtner I: Proteomics of xenografted human breast cancer indicates novel targets related to tamoxifen resistance. Proteomics 6: 1038-1048, 2006.

16. González-Zamorano M, Mendoza-Hernández G, Xolalpa W, Parada C, Vallecillo AJ, Bigi F and Espitia C: Mycobacterium tuberculosis glycoproteomics based on ConA-lectin affinity capture of mannosylated proteins. J Proteome Res 8: 721-733, 2009.

17. Zhang D, Tai LK, Wong LL, Chiu LL, Sethi SK and Koay ES: Proteomic study reveals that proteins involved in metabolic and detoxification pathways are highly expressed in HER-2/neupositive breast cancer. Mol Cell Proteomics 4: 1686-1696, 2005.

18. Chang XZ, Li DQ, Hou YF, Wu J, Lu JS, Di GH, Jin W, Ou ZL, Shen ZZ and Shao ZM: Identification of the functional role of peroxiredoxin 6 in the progression of breast cancer. Breast Cancer Res 9: R76, 2007.

19. Le Naour F, Misek DE, Krause MC, Deneux L, Giordano TJ, Scholl S and Hanash SM: Proteomics-based identification of RS/ DJ-1 as a novel circulating tumor antigen in breast cancer. Clin Cancer Res 7: 3328-3335, 2001.

20. Bianchi MS, Bianchi NO and Bolzán AD: Superoxide dismutase activity and superoxide dismutase-1 gene methylation in normal and tumoral human breast tissues. Cancer Genet Cytogenet 59: 26-29, 1992.

21. Wei L, Liu TT, Wang HH, Hong HM, Yu AL, Feng HP and Chang WW: Hsp27 participates in the maintenance of breast cancer stem cells through regulation of epithelial-mesenchymal transition and nuclear factor-kappa B. Breast Cancer Res 13: R101, 2011.

22. Sims JD, McCready J and Jay DG: Extracellular heat shock protein (Hsp)70 and $\mathrm{Hsp} 90 \alpha$ assist in matrix metalloproteinase-2 activation and breast cancer cell migration and invasion. PLoS One 6: e18848, 2011.

23. Wang LP, Bi J, Yao C, Xu XD, Li XX, Wang SM, Li ZL, Zhang DY, Wang $M$ and Chang GQ: Annexin A1 expression and its prognostic significance in human breast cancer. Neoplasma 57: 253-259, 2010.

24. Porter D, Weremowicz S, Chin K, et al: A neural survival factor is a candidate oncogene in breast cancer. Proc Natl Acad Sci USA 100: 10931-10936, 2003.

25. Sahni A, Arévalo MT, Sahni SK and Simpson-Haidaris PJ: The VE-cadherin binding domain of fibrinogen induces endothelial barrier permeability and enhances transendothelial migration of malignant breast epithelial cells. Int J Cancer 125: 577-584, 2009.

26. Moon HG, Jeong SH, Ju YT, Jeong CY, Lee JS, Lee YJ, Hong SC, Choi SK, Ha WS, Park ST and Jung EJ: Up-regulation of RhoGDI2 in human breast cancer and its prognostic implications. Cancer Res Treat 42: 151-156, 2010. 
27. Steeg PS, De la Rosa A, Flatow U, MacDonald NJ, Benedict M and Leone A: Nm23 and breast cancer metastasis. Breast Cancer Res Treat 25: 175-187, 1993.

28. Thornalley PJ: Protecting the genome: defence against nucleotide glycation and emerging role of glyoxalase I overexpression in multidrug resistance in cancer chemotherapy. Biochem Soc Trans 31: 1372-1377, 2003

29. Knauer M, Cardoso F, Wesseling J, Bedard PL, Linn SC, Rutgers EJ and van't Veer LJ: Identification of a low-risk subgroup of HER-2positive breast cancer by the 70 -gene prognosis signature. $\mathrm{Br} \mathrm{J}$ Cancer 103: 1788-1793, 2010.

30. Toi M, Iwata H, Yamanaka T, et al: Japan Breast Cancer Research Group-Translational Research Group: Clinical significance of the 21-gene signature (Oncotype DX) in hormone receptor-positive early stage primary breast cancer in the Japanese population. Cancer 116: 3112-3118, 2010.

31. Pitkanen-Arsiola T, Tillman J, Gu G, et al: Androgen and antiandrogen treatment modulates androgen receptor activity and DJ-1 stability. Prostate 66: 1177-1193, 2006.

32. Tillman J, Yuan J, Gu G, et al: DJ-1 binds androgen receptor directly and mediates its activity in hormonally treated prostate cancer cells. Cancer Res 67: 4630-4637, 2007.

33. Ranganathan S and Tew KD: Analysis of glyoxalase-I from normal and tumor tissue from human colon. Biochim Biophys Acta 1182: 311-316, 1993.

34. Di Ilio C, Angelucci S, Pennelli A, Zezza A, Tenaglia R and Sacchetta P: Glyoxalase activities in tumor and non-tumor human urogenital tissues. Cancer Lett 96: 189-193, 1995.
35. Davidson SD, Cherry JP, Choudhury MS, Tazaki H, Mallouh C and Konno S: Glyoxalase I activity in human prostate cancer: a potential marker and importance in chemotherapy. J Urol 161: 690-691, 1999

36. Rulli A, Carli L, Romani R, Baroni T, Giovannini E, Rosi G and Talesa V: Expression of glyoxalase I and II in normal and breast cancer tissues. Breast Cancer Res Treat 66: 67-72, 2001.

37. Bair WB III, Cabello CM, Uchida K, Bause AS and Wondra GT: GLO1 overexpression in human malignant melanoma. Melanoma Res 20: 85-96, 2010.

38. Sakamoto H, Mashima T, Kizaki A, Dan S, Hashimoto Y, Naito M and Tsuruo T: Glyoxalase I is involved in resistance of human leukemia cells to antitumor agent-induced apoptosis. Blood 95: 3214-3218, 2000.

39. Santarius T, Bignell GR, Greenman CD, et al: GLO1 - a novel amplified gene in human cancer. Genes Chromosomes Cancer 49: 711-725, 2010.

40. Van der Heiden MG, Cantley LC and Thompson CB: Understanding the Warburg effect: the metabolic requirements of cell proliferation. Science 324: 1029-1033, 2009.

41. Ross JS and Harbeck N: Prognostic and predictive factors overview. In: Molecular Oncology of Breast Cancer. Ross JS and Hortobagyi GN (eds). Jones and Bartlett Publishers, Sudbury, MA, 2005. 